\title{
Gamma-Ray Bursts: Afterglows and Central Engines
}

\author{
K.S. Cheng ${ }^{1}$, T. $\mathrm{Lu}^{2}$ \\ ${ }^{1}$ Department of Physics, University of Hong Kong, Pokfulam Road, Hong Kong, China \\ 2 Department of Astronomy, Nanjing University, Nanjing 210093, China
}

\begin{abstract}
Gamma-ray bursts (GRBs) are most intense transient gamma-ray events in the sky when they are on together with the strong evidences (i.e. the isotropic and inhomogeneous distribution of GRBs detected by BASTE) that they are located at cosmological distances, which make them the most energetic events ever known. For example, the observed radiation energies of some GRBs are equivalent to convert more than one solar mass energy into radiation completely. This is thousand times stronger than that of supernova explosion. Unconventional energy mechanisms and extremely high conversion efficiency for these mysterious events are required. The discovery of host galaxies and association with supernovae in the cosmological distances by the recently launched satellite of BeppoSAX and ground based radio and optical telescopes in GRB afterglow provides further support to the cosmological origin of GRBs and put strong constraints on central engines of GRBs. It is the aim of this article to review the possible central engines, energy mechanisms, dynamical and spectral evolution of GRBs, especially focusing on the afterglows in multi-wavebands.
\end{abstract}

Subject headings: gamma-rays: bursts — shock waves — ISM: jets and outflows — radiation mechanisms: non-thermal 


\section{Introduction}

The nature of gamma-ray burst is still a mystery since its discovery about thirty years ago (Klebesadel, Strong \& Olsen 1973). It has been thought that Galactic magnetized neutron stars are promising candidates to the gamma-ray burst sources because of the spectral features that had been observed in several different early instruments (Mazet et al. 1981; Hunter 1984; Fenimore et al. 1988; Murakami et al. 1988). However, the launch of Compton Gamma-Ray Observatory (CGRO) on April 5, 1991 has led to a new insight to these gamma-ray sources. From the spatial distribution of the gamma-ray bursts observed by the Burst and Transient Source Experiment (BATSE) on board CGRO, it was found that they are more consistent to isotropic distribution, which is difficult to be understood by the galactic neutron star models. In addition, the smaller number of weak sources observed in comparing to that of strong sources can be interpreted as we are observing the boundary of the sources. Many models then have been suggested that these high energy gamma-ray sources may originate from extragalactic region. The isotropy of the sources can then be explained and the deficit of weak sources can be understood. However, most of these cosmological models fail to give a quantitative explanation to the spectral features (lines and continuum) observed in the gamma-ray bursts.

In early 1997, the Italian-Dutch Satellite, BeppoSAX, brought the great break-through by rapid and accurate GRB localization and thus provided a few arcmins (or even smaller) error box. With so small an error box, it identified an X-ray counter-part (now known as Xray afterglow) of GRB 970228 just 8 hours after the $\gamma$-ray trigger (Costa et al. 1997). Several hours later, its optical afterglow was also observed (Groot et al. 1997; van Paradijs et al. 1997). Since then, BeppoSAX has observed more than 20 GRBs, of which almost all exhibited X-ray afterglows. Based on the precise localization, many telescopes observed about a dozen

optical afterglows and about ten radio afterglows. Up to now, people have observed host 
galaxies of more than ten GRBs with large red-shifts, showing them definitely at cosmological distances. These great discoveries lead to rapid developments in GRB studies(van Paradijs et al. 2000). A lot of questions have now been clarified. However, compared with GRB itself, afterglow appears to be simpler and has been known much better. In contrast, the GRB itself, especially its energy source and origin, still keeps to be mysterious.

The main observational facts of GRBs are as follows:

\section{(i)Burst Rate:}

BATSE has detected about one GRB per day from an unpredictable direction in the sky. It is believed that the burst rate could be up to $10^{3}$ bursts per year if the actual solid angle and the sky exposure time are corrected.

\section{(ii)Temporal Properties:}

The majority of GBRs has a very complex temporal structures. Their variability $\delta T$ is significantly shorter than the duration $T$. Typically, $\delta T \sim 10^{-2} T$. $T$ is very short, usually only a few seconds to tens of seconds, but occasionally it could be as long as a few tens of minutes or as short as a few milli-seconds. There seems to be a roughly bimodal distribution of long bursts of $T \geq 2 \mathrm{~s}$ and short bursts of $T \leq 2 \mathrm{~s}$ (Kouveliotou et al. 1993). The time scales of variability $(\delta T)$, especially their rising time, may be as short as only milli-seconds or even sub-milli-seconds. There are various time dilations in temporal profiles of GRBs. If the GBRs are cosmological origin then the temporal profiles and spectra of more distant sources will be time dilated compared to those relatively nearby sources (e.g. Norris et al. 1994; Wei \& Lu 1997). Time dilations in different energy channels were also discovered (e.g. Cheng et al. 1995; 1996b).

\section{(iii)Spatial distribution:}

The angular distribution of GRBs' position on the sky is perfectly isotropic (Greiner 
1999). For the first 1005 BATSE bursts the observed dipole and quadrupole (corrected to BATSE sky exposure) relative to the galaxy are $0.017 \pm 0.018$ and $-0.003 \pm 0.009$ respectively. These values are $0.9 \sigma$ and $0.3 \sigma$ deviated from complete isotropy (Meegan et al. 1992; Briggs 1995) respectively. This distribution favors GRBs at cosmological distances at least statistically. However, GRBs at extended dark halo of our Galaxy could also explain this feature. This led to a great debate between galactic origin and cosmological origin. There are a few strong bursts and many more weak bursts. A sample of the first 601 bursts is used to analyze the distribution of sources in space and shows a $14 \sigma$ deviated from the homogeneous flat space distribution (Pendleton et al.1995) but compatible with a cosmological distribution. However, the distribution of short bursts is not inconsistent with homogeneous Euclidian distribution (Katz \& Canel 1996).

(iv)Total Energy of the Burst: The $\gamma$-ray fluences are typically in the range of $(0.1-10) \times 10^{-6} \mathrm{ergs} / \mathrm{cm}^{2}$. If the possible beaming is ignored, these fluences imply that the total burst energies are $10^{45}-10^{46} \mathrm{ergs}$ for galactic halo distance and $10^{52}-10^{53} \mathrm{ergs}$ for cosmological distance. However, the energy release of GRB 990123 in $\gamma$-rays is already as large as about $3.4 \times 10^{54} \mathrm{ergs}$, if considering the low efficiency of $\gamma$-rays radiation, the total energy release could be a few times of $10^{55}$ ergs or even much larger, this imposes strong challenge for all GRB models(Kulkarni et al. 1999a; Andersen et al. 1999).

\section{(v)Spectral properties:}

Most of GRB power is radiated in the 100 - $1000 \mathrm{keV}$ range, but photons up to $18 \mathrm{GeV}$ or down to a few keV have also been registered. The non-thermal spectra of GRBs are best fitted by a broken power-law with two spectral indices. If a simple power-law fit is used, namely $\mathrm{F}\left(E_{\gamma}\right) \propto E_{\gamma}^{-\alpha}$, where $\alpha$ is about $1.8-2$ (Piran 1999). The low energy part of the

spectrum behaves in many cases like a simple power-law: $\mathrm{F} \propto E_{\gamma}^{-\beta}$ with $-1 / 2<\beta<1 / 3$ (Katz 1994; Cohen et al. 1997). This can be easily explained in terms of the non-uniform 
magnetic distribution inside the fireball shock front (Cheng \& Wei 1996). Observations by earlier detectors as well as by BATSE have shown that the spectrum varies during the bursts. Most bursts evolve from hard to soft, but different trends were found (Norris et al. 1986; Ford et al. 1995). Both absorption and emission features had been reported by various experiments prior to BATSE. Absorption lines in the 20 - $40 \mathrm{keV}$ range had been observed by several experiments (Murakami et al. 1988; Fenimore et al.1988), but never simultaneously. Emission features near $400 \mathrm{keV}$ had been claimed in other bursts (Mazets et al. 1980). However BATSE has not found any of the spectral features (Palmer et al. 1994; Band et al. 1996).

\section{(vi)Afterglows:}

Afterglows are counterparts of GRBs at wave bands other than $\gamma$-rays, may be in Xray, optical, or even radio bands (Costa et al. 1997; van Paradijs et al. 1997; Galama et al. 1997; Frail et al. 1997; Taylor et al. 1997; Piran 1999). They are variable, typically decaying according to power laws: $F_{\nu} \propto t^{-\alpha}(\nu=\mathrm{X}$, optical, ......) with $\alpha=1.1-1.6$ for X-ray, $\alpha=1.1-2.1$ for optical band. X-ray afterglows can last days or even weeks; optical afterglows and radio afterglows months. The most important discovery is that many afterglows show their host galaxies being definitely at cosmological distances (with large redshifts up to $Z=3.4$ or even 5). Thus, the debate is settled down, GRBs are at cosmological distances, they should be the most energetic events ever known since the Big Bang.

\section{The Standard Fireball Shock Model}

\section{(i)Stellar Level Event:}

The variability time scale is usually very short. Let $\delta T \sim \mathrm{ms}$, then, the space scale of the initial source, $R_{\mathrm{i}}<c \delta T \sim 3 \times 10^{2} \mathrm{~km}$. Hence, even for black hole, considering $R=2 \mathrm{G} M / c^{2}$, 
we have $M \leq c^{3} \delta T /(2 \mathrm{G}) \sim 10^{2} \mathrm{M}_{\odot}$. If the burster is not a black hole, its mass should be much smaller. Thus, we can conclude that the GRB should be a stellar phenomenon and the burster should be a compact stellar object which may be related with neutron star (or strange star) or stellar black hole.

\section{(ii)Fireball:}

¿From the measured fluence $F$ and the measured distance $D$, if emission is isotropic, we can calculate the total radiated energy to be $E_{0}=F\left(4 \pi D^{2}\right) \approx 10^{51}\left(F /\left(10^{-6} \mathrm{ergs} / \mathrm{cm}^{2}\right)\right)(D /(3 \mathrm{Gpc}))^{2}$. Thus, very large energy $\left(10^{51} \mathrm{ergs}\right)$ is initially contained in a small volume of $(4 / 3) \pi R_{i}^{3} \sim$ $1 \times 10^{23} \mathrm{~cm}^{3}$. This should be inevitably a fireball, of which the optical depth for $\gamma \gamma \longrightarrow \mathrm{e}^{+} \mathrm{e}^{-}$, $\tau_{\gamma \gamma}$, is very large. Consider a typical burst its average optical depth can be written as:

$$
\tau_{\gamma \gamma}=\frac{f_{\mathrm{p}} \sigma_{\mathrm{T}} F D^{2}}{R_{\mathrm{i}}^{2} m_{\mathrm{e}} c^{2}} \sim 10^{17} f_{\mathrm{p}}\left(\frac{F}{10^{-6} \mathrm{ergs} / \mathrm{cm}^{2}}\right)\left(\frac{D}{3 \mathrm{Gpc}}\right)^{2}\left(\frac{\delta T}{1 \mathrm{~ms}}\right)^{-2},
$$

where $f_{\mathrm{p}}$ denotes the fraction of photon pairs satisfying $\sqrt{E_{1} E_{2}}>m_{\mathrm{e}} c^{2}$.

For so large an optical depth, there seem to appear two serious difficulties. First, the radiation in an optically thick case should be thermal, while the observed radiation is definitely non-thermal. Second, high energy photons should be easily converted into $\mathrm{e}^{+} \mathrm{e}^{-}$ pairs, while the observed high energy tail indicates that this convertion has not happened. However, it is very interesting to note that just such a large optical depth paves the way to solve both of them.

\section{(iii)Compactness Problem:}

In fact, the luminosity of the thermal radiation, according to the Stefan-Boltzmann law, should be proportional to the surface of the fireball which is initially so small that the thermal radiation can not be observed. However, just due to the large optical depth, the radiation pressure should be very high and could accelerate the fireball expansion to become ultra-relativistic with a large Lorentz factor $\gamma$. After expanding to a large enough 
distance, it may be getting optically thin. At this time, the non-thermal $\gamma$-ray bursts can be observed. Does such a large distance contradict the compactness relation $R_{\mathrm{i}} \leq \mathrm{c} \delta T$ compared with the milli-second variabilities? To answer this question, let us first note that this relation holds only for non-relativistic (rest) object with $R_{\mathrm{i}}$ denoting its space scale. For an ultra-relativistically expanding fireball, the compactness relation should be relaxed to

$$
R_{\mathrm{e}} \leq \gamma^{2} \mathrm{c} \delta T
$$

here $R_{\mathrm{e}}$ is the space scale of the expanding fireball with Lorentz factor $\gamma$. Considering two photons we observed at two different times apart by $\delta T$, as the emitting region is moving towards the observer with a Lorentz factor $\gamma \gg 1$, the second photon should be emitted at a far nearer place than the first one. This gives effectively short time variabilities and leads to the additional factor $\gamma^{2}$ appearing in the above compactness relation.

The factor $f_{\mathrm{p}}$ in the optical depth $\tau_{\gamma \gamma}$ also sensitively depends on the ultra-relativistic expansion of the fireball. As for this case, the observed photons are blue-shifted, in the comoving frame, their energy should be lower by a factor of $\gamma$, and fewer photons will have sufficient energy to produce pairs. This gives a factor depending on spectral index $\alpha$, namely a factor of $\gamma^{2 \alpha}$ in $\tau_{\gamma \gamma}$.

\section{(iv) Ultra-relativistic expansion:}

Therefore, the optical depth $\tau_{\gamma \gamma}$ will decrease by a factor of $\gamma^{4+2 \alpha}$ for the ultra-relativistically expanding fireball (Goodman 1986; Paczyński 1986; Piran 1999; Krolik \& Pier 1991):

$$
\tau_{\gamma \gamma}=\frac{f_{\mathrm{p}}}{\gamma^{2 \alpha}} \frac{\sigma_{\mathrm{T}} F D^{2}}{R_{\mathrm{e}}^{2} \mathrm{~m}_{\mathrm{e}} \mathrm{c}^{2}} \approx \frac{10^{17}}{\gamma^{(4+2 \alpha)}} f_{\mathrm{p}}\left(\frac{F}{10^{-6} \mathrm{ergs} / \mathrm{cm}^{2}}\right)\left(\frac{D}{3 \mathrm{Gpc}}\right)^{2}\left(\frac{\delta T}{1 \mathrm{~ms}}\right)^{-2} .
$$

Note, the spectral index $\alpha$ is approximately 2 , we will have $\tau_{\gamma \gamma}<1$ for $\gamma>10^{17 /(4+2 \alpha)} \sim 10^{2}$. Thus, in order for the fireball to become optically thin, as required by the observed nonthermal spectra of $\gamma$-ray bursts, its expanding speed should be ultra-relativistic with Lorentz 
factor

$$
\gamma>\sim 10^{2}
$$

This is a very important character for GRBs, which limits the baryonic mass contained in the fireball seriously. If the initial energy is $E_{0}$, then the baryonic mass $M$ should be less than

$$
E_{0} /\left(\mathrm{c}^{2} \gamma\right) \approx 10^{-5} \mathrm{M}_{\odot}\left(E_{0} /\left(2 \times 10^{51} \mathrm{ergs}\right)\right)
$$

otherwise, the initial energy can not be converted to the kinetic energy of the bulk motion of baryons with such a high Lorentz factor. Most models related with neutron stars contain baryonic mass much higher than this limit. This is the famous problem named as "baryon contamination".

It is worthwhile to note that this very condition $\gamma>\sim 10^{2}$ can also explain the existence of the high energy tail in the GRB spectra, as the observed high energy photons should be only low energy photons in the frame of emitting region, they are not energetic enough to be converted into $\mathrm{e}^{+} \mathrm{e}^{-}$pairs.

\section{(v)Internal-external shock:}

What is the radiation mechanism in the fireball model? The fireball expansion has successfully made a conversion of the initial internal energy into the bulk kinetic energy of the expanding ejecta. However, this is the kinetic energy of the associated protons, not the photons. We should have another mechanism to produce radiation, otherwise, even after the fireball becoming optically thin, the $\gamma$-ray bursts can not be observed. Fortunately, the shocks described below can do such a job.

The fireball can be regarded as roughly homogeneous in its local rest frame, but due to the Lorentz contraction, it looks like a shell (ejecta) with width of the initial size of the

fireball. As the shell collides with inter-stellar medium (ISM), shocks will be produced (Rees \& Mészáros 1992; Katz, J., 1994; Sari \& Piran 1995; Mitra 1998). These are usually called as 
external shocks. Relativistic electrons that have been accelerated in the relativistic shocks will usually emit synchrotron radiation. As the amount of swept-up interstellar matter getting larger and larger, the shell will be decelerated and radiation of longer wave length will be emitted. Thus, an external shock can produce only smoothly varying time-dependent emission, not the spiky multi-peaked structure found in many GRBs. If the central energy source is not completely impulsive, but works intermittently, it can produce many shells (or many fireballs) with different Lorentz factors. Late but faster shells can catch up and collide with early slower ones, and then, shocks (internal shocks) thus produced will lead to the observed bursting $\gamma$-ray emission (Rees \& Mészáros 1994; Paczyński \& Xu 1994). This is the so called internal-external shock model, internal shocks give rise to $\gamma$-ray bursts and external shocks to afterglows. The internal shocks can only convert a part of their energies to the $\gamma$-ray bursts, other part remains later to interact with the interstellar medium and lead to afterglows. Typically, the GRB is produced at a large distance of about $10^{13} \mathrm{~cm}$ to the center, such a large distance is allowed according to the relaxed compactness relation $R_{\mathrm{e}} \leq \gamma^{2} \mathrm{c} \delta T$, while its afterglows are produced at about $10^{16} \mathrm{~cm}$ or even much farther. This internal-external shock scenario, under the simplified assumptions of uniform environment with typical ISM number density of $n \sim 1 \mathrm{~cm}^{-3}$, isotropic emission of synchrotron radiation and only impulsive energy injection, is known as the standard model.

\section{(vi)Spectra of afterglows:}

The instantaneous spectra of afterglows, according to this model, can be written as $F_{\nu} \propto \nu^{\beta}$, with different $\beta$ for different range of frequency $\nu$ (Sari et al. 1998; Piran 2000). Let $\nu_{s a}$ be the self absorption frequency, for which the optical depth $\tau\left(\nu_{s a}\right)=1$. For $\nu<\nu_{s a}$, we have the Wien's law: $\beta=2$. For $\nu_{s a}<\nu<\min \left(\nu_{\mathrm{m}}, \nu_{\mathrm{c}}\right)$, we can use the low energy synchrotron tail, $\beta=-1 / 3$. Here $\nu_{\mathrm{m}}$ is the synchrotron frequency of an electron with characteristic energy, $\nu_{\mathrm{c}}$ is the cooling frequency, namely the synchrotron frequency of an 
electron that cools during the local hydrodynamic time scale. For frequency within $\nu_{\mathrm{m}}$ and $\nu_{\mathrm{c}}$, we have $\beta=-1 / 2$ for fast cooling $\left(\nu_{\mathrm{c}}<\nu_{\mathrm{m}}\right)$ and $\beta=-(p-1) / 2$ for slow cooling $\left(\nu_{\mathrm{m}}<\nu_{\mathrm{c}}\right)$. For $\nu>\max \left(\nu_{\mathrm{m}}, \nu_{\mathrm{c}}\right)$, we have $-p / 2$. Here, $p$ is the spectral index of the emitting electrons: $N(E) \propto E^{-p}$.

\section{Dynamical Evolution of the Fireball}

During the $\gamma$-ray bursting phase and the early stage of afterglows, the fireball expansion is initially ultra-relativistic and highly radiative, but finally it would be getting into nonrelativistic and adiabatic, a unified dynamical evolution should match all these phases. In fact, the initial ultra-relativistic phase has been well described by some simple scaling laws (Mészáros \& Rees 1997a; Vietri 1997; Waxman 1997a; Wijers et al. 1997), while the final non-relativistic and adiabatic phase should obey the Sedov (1969) rule, which has well been studied in Newtonian approximation. The key equation (Blandford \& McKee 1976; Chiang \& Dermer 1999) is

$$
\frac{\mathrm{d} \gamma}{\mathrm{d} m}=-\frac{\gamma^{2}-1}{M},
$$

here $m$ denotes the rest mass of the swept-up medium, $\gamma$ the bulk Lorentz factor, and $M$ the total mass in the co-moving frame including internal energy $U$. This equation was originally derived under the ultra-relativistic condition. The widely accepted results derived under this equation are correct for ultra-relativistic expansion. Accidentally, these results are also suitable for the non-relativistic and radiative case. However, for the non-relativistic and adiabatic case, they will lead to wrong result " $v \propto R^{-3 "}$ ( $v$ is the velocity), while the correct Sedov result should be " $v \propto R^{-3 / 2}$ ", as first pointed out by Huang, Dai \& Lu (1999a,b).

It has been proved (Huang, Dai \& Lu 1999a,b) that in the general case, the above 
equation should be replaced by

$$
\frac{\mathrm{d} \gamma}{\mathrm{d} m}=-\frac{\gamma^{2}-1}{M_{e j}+\epsilon m+2(1-\epsilon) \gamma m},
$$

here $M_{e j}$ is the mass ejected from GRB central engine, $\epsilon$ is the radiated fraction of the shock generated thermal energy in the co-moving frame. The above equation will lead to correct results for all cases including the Sedov limit. This generic model is suitable for both ultra-relativistic and non-relativistic, and both radiative and adiabatic fireballs. As proved by Huang et al. (1998a,b), Wei \& Lu (1998a) and Dai et al. (1999a), only several days after the burst, a fireball will usually become non-relativistic and adiabatic, while the afterglows can last observable for some months, the above generic model is really useful and important.

\section{Comparison and Association of GRB with SN}

Supernova was known as the most energetic phenomenon at the stellar level. SN explosion is the final violent event in the stellar evolution. Dynamically, it can also be described as a fireball, which however expands non-relativistically. After the SN explosion, there is usually a remnant which can shine for more than thousands of years and be well described dynamically by Sedov model (Sedov 1969).

GRB is also a phenomenon at the stellar level. However, it is much more energetic and much more violent than SN explosion! It has been proved to be described as a fireball, which expands ultra-relativistically. The GRB may also leave a remnant which shines for months now known as afterglow.

Their comparison is given in Table I: 
Table I

\begin{tabular}{lll}
\hline & GRBs & SNe \\
\hline Burst & Bursting $\gamma$-rays & SN explosion \\
\hline Energy up to & $10^{54}$ ergs & $10^{51} \mathrm{ergs}$ \\
Time Scale & $10 \mathrm{sec}$ & Months \\
Profile & irregular & smooth \\
Wave Band & $\gamma$-ray & Optical \\
\hline Relic & Afterglow & Remnant \\
\hline Time Scale & Months & $10^{3}$ Years \\
Wave Band & Multi-band & Multi-band \\
\hline Understanding & & \\
\hline Fireball Expansion & Ultra-relativistic & Non-relativistic \\
Mechanism & $? ? ?$ & Stellar Core Collapse \\
Key Process & $? ? ?$ & Neutrino process \\
\hline
\end{tabular}

In April 1998, a SN 1998bw was found to be in the 8' error circle of the X-ray afterglow of GRB 980425 (Galama et al. 1998; Kulkarni et al. 1998). However, its host galaxy is at a red-shift $\mathrm{z}=0.0085$ (Tinney et al. 1998), indicating a distance of $38 \mathrm{Mpc}$ (for $H_{0}=65 \mathrm{~km}$ $\mathrm{s}^{-1} \mathrm{Mpc}^{-1}$ ), which leads the energy of the GRB to be too low, only about $5 \times 10^{47}$ ergs, 4 orders of magnitude lower than normal GRB.

Later, in the light curves of GRB 980326 (Bloom et al. 1999; Castro-Tirado \& Gorosabel 1999b) and GRB 970228 (Reichart 1999; Galama et al. 2000), some evidence related with SN was found. This is a very important question worth while to study further (see e.g. Wheeler 1999). These two violent phenomena, GRB and SN, might be closely related. They might be just two steps of one single event (Woosley et al. 1999; Cheng \& Dai 1999; Wang et al. 2000b; Dai 1999d). It is interesting to note that the first step might provide a low 
baryon environment for the second step to produce GRB. Such a kind of models can give a way to avoid the baryon contamination.

\section{Post-Standard Effects}

The standard model described above is based on the following simple assumptions: (1) the fireball expanding relativistically and isotropically; (2) impulsive injection of energy from inner engine to the fireball(s); (3) synchrotron radiation as the main radiation mechanism; (4) uniform environment with typical particle number density of $n=1 \mathrm{~cm}^{-3}$. This model is rather successful in that its physical picture is clear, results obtained are simple, and observations on GRB afterglows support it at least qualitatively but generally. However, various quantitative deviations have been found. Thus, the simplifications made in the standard model should be improved. These deviations may reveal important new information. In the following, we will discuss some of the effects due to these deviations (the post-standard effects) (Lu 1999, 2000; Dai 2000e).

\section{(i)Environment effects:}

In the early days after the discovery of afterglows, Dai and $\mathrm{Lu}(1998 \mathrm{c})$ studied the possible non-uniformity of the surrounding medium. They used the general form of $n \propto R^{-k}$ to describe the non-uniform environment number density. By fitting the X-ray afterglow of GRB 970616, they found $k=2$ which is just the form of a wind environment. This indicates that the surrounding medium of GRB 970616 was just a stellar wind. After the detailed studies by Chevalier and $\operatorname{Li}(1999,2000)$, the stellar wind model for the environment of GRBs has now become widely interested. As the properties of GRBs' environment contain

important information related with their pregenitors, this stellar wind model provides strong support to the view of massive star origin of GRBs. 
Another environment effect is due to the deviation from the standard number density of $n=1 \mathrm{~cm}^{-3}$. Some afterglows of GRBs show that their lightcurves obey a broken power law. For example, according to Fruchter et al.(1999), the optical lightcurve of GRB 990123 shows a break after about two days, its slope being steepened from -1.09 to -1.8 . Dai and Lu (1999b) pointed out that a shock undergoing the transition from a relativistic phase to a nonrelativistic phase may show such a break in the light curve. If there are dense media and/or clouds in the way, this break may happen earlier to fit the observed steepening. Recently, Wang, Dai \& Lu (2000a) proved that the dense environment model can also explain well the radio afterglow of GRB 980519 (Frail et al. 2000).

\section{(ii) Additional energy injection:}

Lightcurves of some optical afterglows even show the down-up-down variation such as GRB 970228 and 970508. These features can be explained by additional long time scale energy injection from their central engines (Dai \& Lu 1998a, b; Rees \& Mészáros 1998; Panaitescu et al. 1998). In some models, a millisecond pulsar with strong magnetic field can be produced at birth of a GRB. As the fireball expands, the central pulsar can continuously supply energy through magnetic dipole radiation. Initially, the energy supply is rather small, the afterglow shows declining. As it becomes important, the afterglow shows rising. However, the magnetic dipole radiation should itself attenuate later. Thus, the down-up-down shape would appear naturally. Dai \& Lu (2000a) further analysed GRB 980519, 990510 and 980326 , with dense environment also being taken into account, and found results agreeing well with observations. Wang \& Dai (2000e) further considered both homogeneous and wind external media, the R-band light curve of GRB 000301c was also well fitted.

Recently, the GRB 000301c afterglow shows three break appearance in the R-band light curve, and extremely steep decay slope -3.0 at late time. This unusual afterglow can be explained by assuming more complicated additional energy injections and dense medium 
(Dai \& Lu 2000b).

\section{(iii) Additional radiations:}

Though synchrotron radiation is usually thought to be the main radiation mechanism, however, under some circumstances, the inverse Compton scattering may play an important

role in the emission spectrum, and this may influence the temporal properties of GRB afterglows (Wei \& Lu 1998a, b, 2000a). Wang, Dai \& Lu (2000c) even consider the inverse Compton scattering of the synchrotron photons from relativistic electrons in the reverse shock. Under appropriate physical parameters of the GRBs and the interstellar medium, this mechanism can excellently account for the prompt high energy gamma-rays detected by EGRET, such as from GRB 930131. It is interesting to note that during the GRB phase, not only electromagnetic radiations, but also neutrinos, will be emitted (Waxman \& Bahcall 1997, 2000; Halzen 1998; Dai \& Lu 2000c). The prompt neutrino emission from reverse shocks as a result of the interaction of relativistic fireballs with their surrounding wind pointed out by Dai \& Lu (2000c) may be very important.

Later data in the afterglows of GRB 970228 (Reichart 1999; Galama et al. 2000) and 980326 (Bloom et al. 1999; Castro-Tirado \& Gorosabel 1999b) may show the deviations as additional contributions from supernovae.

\section{(iv)Beaming effects:}

GRB 990123 has been found very strong in its $\gamma$-ray emission, and the red shift of its host galaxy is very large $(\mathrm{z}=1.6)$ (Kulkarni et al. 1999a; Galama et al. 1999; Akerlof et al. 1999; Castro-Tirado, et al. 1999a; Hjorth, et al. 1999; Andersen, et al. 1999). If its radiation is isotropic, the radiation energy only in $\gamma$-rays is already as high as $E_{\gamma} \sim 3.4 \times 10^{54}$ ergs, closely equals two solar rest energy $\left(E_{\gamma} \approx 2 \mathrm{M}_{\odot} \mathrm{c}^{2}\right)$ ! As the typical mass of the stellar object is in the order of $\sim 1 \mathrm{M}_{\odot}$, while the radiation efficiency for the total energy converting 
into the $\gamma$-ray emission is usually very low, such a high emission energy is very difficult to understand (Wang, Dai \& Lu 2000d).

As some GRBs showed their isotropic radiation energy to be as high as $\sim \mathrm{M}_{\odot} \mathrm{c}^{2}$, this has been regarded as an energy crisis. A natural way to relax this crisis is to assume that the radiation of GRB is jet-like, rather than isotropic. Denote the jet angle as $\Omega$, then the radiation energy $E$ will be reduced to $E \Omega / 4 \pi$. At the same time, the estimated burst rate should increase by a factor of $4 \pi / \Omega$. However, we should find out its observational evidences. Rhoads $(1997,1999)$ analysed this question, and predicted that the sideways expansion in jet-like case will produce a sharp break in the GRB afterglow light curves (see also Pugliese et al. (2000), Sari et al. (1999) and Wei \& Lu (2000b, c)). Kulkarni et al. (1999c) regarded the break in the light curve of GRB 990123 as the evidence for jet. However, Panaitescu \& Mészáros (1999), Moderski, Sikora \& Bulik (2000) performed numerical calculation and denied the appearence of such a sharp break. Wei \& Lu (2000b) re-analysed the dynamical evolution of the jet blast wave and found that a sharp break can only exist in the case of extremely small beaming angle. Huang et al. (2000a, b) made a detailed calculation and proved that the breaks in the lightcurves are mainly due to the relativistic to non-relativistic transition, not due to edge effect and lateral expansion effect of the jet, and may appear only for small electron energy fraction and small magnetic energy fraction. However, they stressed that the afterglows of jetted ejecta can be clearly characterized by rapid fading in the non-relativistic phase with index $\alpha \geq 2.1$ (Huang et al 2000c). Recently, Dai, Huang and $\mathrm{Lu}(2000 \mathrm{f})$ pointed out that the effect of dust extinction on jetted GRB afterglows may obviously enhance the breaak in their light curves.

Gou et al. (2000) used a set of refined dynamical equations and a realistic lateral speed of the jet, calculated the evolution of a highly collimated jet that expands in a stellar wind environment and the expected afterglow from such a jet. They found that in the wind 
environment, no obvious break will appear even at the time when the blast wave transits from the relativistic phase to the non-relativistic phase, and there will be no flattening tendency even up to $10^{9} \mathrm{~s}$. Further calculations on the anisotropic jets expanding in different kinds of wind have also been made (Dai \& Gou 2000d). GRB 991208 was argued to arise from a highly anisotropic jet expanding in the wind of a red supergiant.

\section{Central Engines and Energy Sources}

It is commonly agreed that the nature of the central engine, which powers the fireball into space, creates shocks where burst of gamma-ray emission and the associated afterglow are emitted, is the most difficult part of GRB-modelling. In fact there is no consensus on the GRB central engine even after the discovery of the afterglow. The main reason is that most observed properties of GRBs are emitted from regions far away from the central engine. Since there are less constraints in the central engine models, it is not surprised to find that there are over hundred proposed central engine models in the literatures. Here we can only introduce popular models and some selected possible models.

\subsection{Popular Cosmological Central Engine Models}

Although there are no compelling direct observed facts to pin-point what are the central engine, there are number of evidence including energy budget, beaming, host galaxies, association with the star formation region (Hogg \& Fruchter 1999) and association with the

type Ic supernova (Galama et al. 1998) to constraint the possible central engine models. The following models are widely quoted in the GRB papers:

\section{(i)Merger of Two Compact Objects.}


These two compact object systems could be neutron star-neutron star, neutron starblack hole, neutron star-white dwarf. Binary neutron star mergers are believed to be the most possible merger model for GRB (e.g. Paczyński 1986; Eichler et al 1989; Dermer 1992; Narayan, Piran and Shemi 1991; Mao and Paczyński 1992; Davies et al. 1994; Ruffert \& Janka 1999). There are three such binary systems observed in our galaxy (Manchester 1999) and there should be at least 30 because of the beaming effect. Their orbital periods are decreasing and should merge in the time scale of $10^{8}$ years (e.g. Taylor \& Weisberg 1989). This gives the merger rate about $10^{6}-10^{7}$ years per galaxy and consistent with the observed GRB rate (Narayan, Piran \& Shemi 1991). It has been suggested that neutron star-black hole is the more common binary system than neutron star-neutron star system, therefore GRBs result from the merger of these systems (Bethe \& Brown 1998). However, none of such system has been found in our galaxy. After merger, a $2-4 M_{\odot}$ black hole surrounded by a $0.1-0.2 M_{\odot}$ thick accretion disk is formed. The black hole can accrete $0.1 M_{\odot}$ from the disk in a time scale of $10-100$ seconds and the gravitational energy of the accreted matter can provide the energy of GRB process. However, GRB 990123 (Kulkarni et al. 1999a; Andersen et al. 1999) requires $E_{\text {iso }}$ must be up to a few times $10^{55}$ ergs for isotropic emission. This causes problem for the merger models. If anisotropic emission with a beaming factor of $\Delta \Omega / 4 \pi \sim 0.01$ is assumed, this energy can be reduced to $E_{\text {jet }} \sim$ a few times $10^{53}$ ergs.

\section{(ii)Failed Supernova or Collapsar of Hypernova}

These models propose that massive stars can collapse to form a rapidly spinning black hole surrounded by a thick torus (Woosley 1993; Paczyński 1998). Instead of using the gravitational energy of the accreted matter, these model suggest that the main energy reservoir is the rotation energy of the black hole, which can be extracted via the Blandford-Znajek mechanism (Blandford \& Znajek 1977). In these models, the accretion rate is at least over

million time of Eddington rate and the disk magnetic field must be over $10^{15}$ Gauss. These 
models are naturally related to supernova, star formation regions, where massive stars are commonly found, and have enough energy budget for the GRB. On the other hand, these central engines are located in the environment filled with baryonic matter. How can a relativistic fireball be developed? It appears to be a problem. Perhaps, a two-step mechanism can resolve this problem. Cheng and Dai (2000) have proposed a two-step model for GRBs associated with supernovae. In the first step, the core collapse of a star with mass $\geq 19 M_{\odot}$ leads to a massive neutron star and a normal supernova, and subsequently hypercritical accretion of the neutron star from the supernova ejecta may give rise to a jet through neutrino annihilation and Poynting flux along the stellar rotation axis. However, because of too much surrounding matter, this jet rapidly enters a non-relativistic phase and evolves to a large bubble. In the second step, the neutron star promptly implodes to a rapidly rotating black hole surrounded by a torus once the mass of the star increases to the maximum mass, and meanwhile its rotation frequency increases to the upper limit due to the accreted angular momentum. The gravitational binding energy of the torus may be dissipated by a magnetized relativistic wind, which may then be absorbed by the supernova ejecta, thus producing an energetic hypernova. The rotational energy of the black hole may be extracted by the Blandford-Znajek mechanism, leading to another jet. This jet is relatively free of baryons and thus may be accelerated to an ultrarelativistic phase because the first jet has pushed out of its front matter and left a baryon-free exit. Therefore the second jet generates a long/hard GRB and its afterglow. This is because the energy release timescale in the second step is about $E_{\text {rot }} / P_{\mathrm{BZ}} \sim 10^{2} \mathrm{~s}$, which corresponds to the GRB duration, if the involved magnetic field has a strength of the order of $10^{16} \mathrm{G}$, and also because the Lorentz factor of the second jet in this model may be larger than 100 so that photons radiated from this jet can be blue-shifted to hard ones. Recently, some authors (Cen 1998; Wang \& Wheeler 1998) envisioned, in a small cone around some special axis of a newborn neutron star, the matter is assumed to be preferentially first blown out in order to avoid too many baryons 
contaminating a subsequently resulting jet. Our model may provide a plausible way of how such an empty cone is produced: neutrinos from the hypercritical accretion disk annihilate to electron/positron pairs which form the first jet to push its front baryons and leave an exit for the second jet. Therefore, this model can avoid the baryon contamination problem.

\subsection{Possible Cosmological Central Engine Models}

\section{(i)Rapidly Spinning and Strongly Magnetized Compact Objects}

The required energy budget of a typical GRB is $\sim 10^{53} \mathrm{ergs}$, which is about the rotational energy of a neutron star rotating in its maximum Keplerian speed. The main problem is how to release this amount of rotation energy in a time scale of $10-100$ seconds, which can be achieved if the star has a super-strong magnetic field ( $\geq 10^{15}$ Gauss). High potential drop ( $\sim 10^{20}$ Volts $)$ can easily produce a relativistic pulsar wind and subsequent radiation can produce the observed GRB spectral properties (Usov 1992; Duncan \& Thompson 1992). The recent discovery of Anomaly X-ray Pulsars and Soft Gamma-ray Repeaters, which are found to be neutron stars with magnetic field strength from $10^{13}-10^{15}$ Gauss, provide support for these models. However, these models exist number of disadvantages because the gamma-rays are expected to be emitted in the internal shocks, which requires that the energy release cannot be continuous and some baryons $\left(\leq 10^{-5} M_{\odot}\right)$ must be there. In fact, these requirements are also important to explain the time variability observed in GRBs. It has been suggested that the super-strong magnetic field is produced after the neutron star is formed. The possible magnetic field production dynamo results from the differential rotation of different parts of the star. If this is the case, an even stronger toroidal field $\sim 10^{17} \mathrm{G}$ can be produced by differential rotation. When the toroidal field generated inside the star becomes strong enough to break the crust, the emerging field together with the broken platelets can be reconnected on the surface of neutron stars and transfer the 
magnetic energy to baryonic matter in the broken platelets. This process can continue until all rotation energy is converted into magnetic energy, which eventually goes to the fireball( Kluźniak \& Ruderman 1998; Ruderman, Tao \& Kluźniak 2000). It was immediately realized that such process could also work for rotating strange stars (Dai \& Lu 1998), who pointed out that the rise of the afterglow in GRB 970508 could result from the continuous energy supply of the central pulsar via pulsar wind.

\section{(ii)Phase Transition of Compact Objects}

Pion condensation in the core of neutron star has been proposed to be the possible mechanism as the energy source of GRBs (e.g. Ramaty et al. 1980). However, it has been shown that such process cannot be a sudden process due to the conservation of charge and baryon number (Glendenning 1992). On the other hand, when a neutron star in Low Mass X-ray Binary accretes over about half a solar mass from its companion, it can undergo a phase transition from neutron star to strange star and release even larger amount of energy to account for GRB. $20-30 \mathrm{MeV}$ is released per baryon during the phase transition. Total energy released this way can be up to about $(4-6) \times 10^{52}$ ergs. Strange star is the stellar object in the quark level. Whether it exists or not is a fundamental physical/astrophysical problem. Its main part is a quark core with large strangeness (known as strange core). There could be a thin crust with mass of only about $\sim\left(10^{-6}-10^{-5}\right) \mathrm{M}_{\odot}($ Alcock et al. 1986; Huang \& Lu 1997a,b; Lu 1997; Cheng et al. 1998), all baryons are contained in the crust. The resulting strange star has a thin crust with mass $\sim 2 \times 10^{-5} M_{\odot}$ and thickness $\sim 150 \mathrm{~m}$, but because the internal temperature is so high $\left(\sim 10^{11} \mathrm{~K}\right)$, the nuclei in this crust may decompose

into nucleons. Approximating strange matter by a free Fermi gas, the total thermal energy of the star is given by $E_{\mathrm{th}} \sim 5 \times 10^{51} \operatorname{ergs}\left(\rho / \rho_{0}\right)^{2 / 3} R_{6}^{3} T_{11}^{2}$, where $\rho$ is the average mass density, $R_{6}$ the stellar radius in units of $10^{6} \mathrm{~cm}$, and $T_{11}$ the temperature in units of $10^{11} \mathrm{~K}$. Adopting $\rho=8 \rho_{0}, R_{6}=1$, and $T_{11}=1.5$, we have $E_{\text {th }} \sim 5 \times 10^{52}$ ergs. The star will cool 
by the emission of neutrinos and antineutrinos, and because of the huge neutrino number density, the neutrino pair annihilation process $\nu \bar{\nu} \rightarrow e^{+} e^{-}$operates in the region close to the strange star surface. The total energy (Haensel, Paczyński \& Amsterdamski 1992) deposited due to this process is $E_{1} \sim 2 \times 10^{48} \operatorname{ergs}\left(T_{0} / 10^{11} \mathrm{~K}\right)^{4} \sim 10^{49} \mathrm{ergs}$ (where $T_{0}$ is the initial temperature) and the timescale for deposition is of the order of $1 \mathrm{~s}$. On the other hand, the processes for $n+\nu_{e} \rightarrow p+e^{-}$and $p+\overline{\nu_{e}} \rightarrow n+e^{+}$play an important role in the energy deposition and the integrated neutrino optical depth (Mészáros \& Rees 1992) due to these processes is $\tau \sim 4.5 \times 10^{-2} \rho_{11}^{4 / 3} T_{11}^{2}$ (where $\rho_{11}$ is the crust density in units of $10^{11} \mathrm{~g} \mathrm{~cm}^{-3}$ ). So the deposition energy is estimated by $E_{2} \sim E_{\text {th }}\left(1-e^{-\tau}\right) \sim 2 \times 10^{52}$ ergs. Here the neutrondrip density $\left(\rho_{11} \sim 4.3\right)$ is used. The process, $\gamma \gamma \leftrightarrow e^{+} e^{-}$, inevitably leads to creation of a fireball. However the fireball must be contaminated by the baryons in the thin crust of the strange star. If we define $\eta=E_{0} / M_{0} c^{2}$, where $E_{0}=E_{1}+E_{2}$ is the initial radiation energy produced $\left(e^{+} e^{-}, \gamma\right)$ and $M_{0}$ is the conserved rest mass of baryons with which the fireball is loaded, then, since the amount of the baryons contaminating the fireball cannot exceed the mass of the thin crust, we have $\eta \geq 5 \times 10^{3}$ and the fireball will expand outward. The expanding shell (having a relativistic factor $\Gamma \sim \eta$ ) interacts with the surrounding interstellar medium and its kinetic energy is finally radiated through non-thermal processes in shocks.

Another possible phase transition model is an accreting strange star collapsing into a naked singularity (Harko \& Cheng 2000).

\section{(iii)Accretion Onto Massive Black Holes}

There are central engine models based on massive black holes associated with Quasars or AGNs in galactic centers (e.g. Carter 1992). These models are ruled out as all GRBs with optical afterglow are not associated with such objects (Piran 1999). Perhaps this conclusion should be revised as all long duration GRBs are not associated with either Quasars or AGNs because all GRBs with afterglow are long duration bursts. Statistical analysis of 
GRBs in the BASTE catalog does show some correlation with certain type of Quasars. For example, Schartel, Andernach \& Greiner (1997) found a surprising evidence of a positional correlation between GRBs and radio-quiet quasars, but for most classes of AGNs, BL Lac objects and radio-loud quasars, no excess coincidences above random expectation; Marani et al (1997) reported a suggestive correlation between GRBs and ACO clusters though some of the significance of correlation can be rather high; Kolatt \& Piran (1996), Hurley et al (1997), and Gorosabel \& Castro-Tirado (1997) studied the angular correlation between GRBs and Abell clusters but obtained different results. There are also many works focused on the studies of GRBs repetition (e.g., Quashnock \& Lamb 1993; Brainerd et al 1995; Meegan et al 1995; Bennett \& Sun 1996) with various statistical methods. But the sound of negative results seems loud.

Recently metal rich Quasars are found to correlate with GRBs in 99\% significant level (Cheng et al. 1997). It can be understood why metal rich Quasars could be the host galaxies of GRBs in the following reasons (Cheng \& Wang 1999). The production rate of compact objects, i.e. neutron stars (NS) and black holes (BH), in active galactic nuclei(AGN) and quasars(QSO), where the frequent supernova explosion is used to explain the high metallicity (Artymowicz, Lin \& Wampler 1993; Zurek et al 1994), is very high due to the interaction between the accretion disk and main sequence stars in the nucleus of the quasar. The compact object-red giant $\operatorname{star}(\mathrm{RG})$ binaries can be easily formed due to the large captured cross-section of the red giant stars. The (NS/BH, NS/BH) binary can be formed after the supernova explosion of the (NS/BH, RG) binary. Intense transient gamma-ray emission (gamma-ray burst) and gravitational radiation can result from the merger of these two compact objects. Collision between helium core (Hc) of RG and black hole may also take place and may also result in long duration gamma-ray bursts but no gravitational waves (Popham, Woosley \& Fryer 1999). The estimated merger rate of (NS/BH, NS/BH) binaries and (Hc, $\mathrm{BH})$ is proportional to the metal abundance $\left(\frac{N V}{C I V}\right)$ and can be as high as $10^{-3}\left(\frac{N V}{C I V} / 0.01\right)$ 
per year per AGN/QSO.

Massive black hole is not favorable central engine because of the short time variability, which suggests that the central engine should be a stellar mass compact object. However, this is misleading because the relativistic motion of the fireball can reduce the time scale in the GRBs (Lu, Cheng, Zhao \& Yang 2000). Cheng and Lu (2001) also suggest that an extreme Kerr black hole with a mass $\sim 10^{6} M_{\odot}$, a dimensionless angular momentum $A \sim 1$ and a marginally stable orbital radius $r_{\mathrm{ms}} \sim 3 r_{\mathrm{s}} \sim 10^{12} M_{6} \mathrm{~cm}$ located in a normal galaxy, may produce a Gamma-ray Burst by capturing and disrupting a star. During the capture period, a transient accretion disk is formed and a strong transient magnetic field $\sim 2.4 \times 10^{9} M_{6}^{-1 / 2}$ $\mathrm{G}$ (Haswell et al. 1992), lasting for $r_{\mathrm{ms}} / c \sim 30 M_{6} \mathrm{~s}$, may be produced at the inner boundary of the accretion disk. A large amount of rotational energy of the black hole is extracted and released in an ultra relativistic jet with a bulk Lorentz factor $\Gamma$ larger than $10^{3}$ via the Blandford-Znajek process. The relativistic jet energy can be converted into $\gamma$-ray radiation via an internal shock mechanism. The GRB duration should be the same as the life time of the strong transient magnetic field. The maximum number of sub-bursts is estimated to be $r_{\mathrm{ms}} / h \sim\left(10-10^{2}\right)$ because the disk material is likely to break into pieces with a size about the thickness of the disk $h$ at the cusp $\left(2 r_{\mathrm{s}} \leq r \leq 3 r_{\mathrm{s}}\right)$. The shortest rise time of the burst estimated from this model is $\sim h / \Gamma c \sim 3 \times 10^{-4} \Gamma_{3}^{-1}(h / r)_{-2} M_{6}$ s. The model gamma-ray burst density rate is also estimated to be consistent with the observed GRB burst rate. Furthermore, the optical afterglow can also be understood in this model. Because in the early stage of forming transient accretion disk, the material supplied by the tidally disrupted star is clumped in the relatively cool disk. This eventually leads to a thermal instability, which results in an increase of the viscosity of the disk. As the instability propagates across the disk, the stability of a time-dependent disk and variability of the mass-deposition rate provide a possible explanation for the behavior of GRB optical afterglow (Lu, Cheng \& Zhao 2000). 


\subsection{Galactic Central Engine Models}

Although the cosmological origin of GRBs are strongly supported by the observation, at least a fraction of GRBs originating from the Galactic neutron stars is also suggested by the observation of absorption lines and soft X-ray emission from some bursts (but BATSE has not seen such line features yet). About $20 \%$ of GRBs in the KONUS catalog show single absorption features at energies between about 20 and $60 \mathrm{keV}$ (Mazets et al. 1981) and three bursts seen by Ginga detector show double absorption features in the same energy range (Murakami et al. 1988). These features are usually attributed to the cyclotron resonant scattering of photons in strong magnetic fields of the order of $\sim 10^{12} G$ near the neutron star surface. Therefore it is reasonable to think that at least a subclass of GRBs may be originated in our Galaxy. The Galactic models also result in an isotropic distribution because the bursts are produced by high velocity neutron stars born in the vicinity of the disk flowing out into the halo (e.g. Li \& Dermer 1992; Podsiadlowski, Rees \& Ruderman 1995; Wei \& $\mathrm{Lu}$ 1996). Observations show that the mean pulsar birth velocity is about $450 \mathrm{~km} \mathrm{~s}^{-1}$ (Lyne \& Lorimer 1994), which provides some support to such models. The energy sources basically are accretion energy, thermonuclear flash, rotation energy and internal energy of neutron stars (for a general review of galactic GRB models cf. Harding 1991). But the most promising models are starquake models. As a neutron star quakes, the oscillation of the magnetic field lines anchored in the crustal platelet produces Alfven wave. Blaes et al. (1989) have argued that such Alfven wave can accelerate charged particles and produce GRBs. However, the question is can these high velocity neutron stars constitute a subclass of observed GRBs? Hartmann \& Narayan (1996) considered the global energy requirements of such models and argued that the rotational energy of neutron stars is insufficient by, at least, orders of magnitude to provide the observed burst rate because the origin of the starquake energy comes from the crustal superfluid, which is only $1 \%$ of the stellar rotation energy. Wei and Cheng (1997) also carried a statistical analysis by using the observed period 
distribution of pulsars and concluded the same result. On the other hand it is possible to make use of full rotational energy, if pulsar activities can be re-ignited in these old neutron stars (Ruderman \& Cheng 1988; Cheng \& Ding 1993; Ding \& Cheng 1997). It is suggested that the detail study of GRB spectral features at lower and higher energy components can be a possible way to distinguish between cosmological and Galactic origin of GRBs (Wei \& Cheng 1997).

\section{Outlook}

Indeed the discovery of the red-shifts of the host galaxies of GRBs in afterglows is a major breakthrough in understanding the nature of GRBs (van Paradijs et al. 2000). The subsequent study of spectral evolution of afterglows in terms of fireball models reveals many important features of the environment of GRBs. The standard internal-external shock model, which is built under many simplifications, has been proved to be well fitted by observations qualitatively but generally. Based on the success of this model, it should be very important to study the deviations from the standard model, which indicate that the simplifications should be relaxed in some aspects. Hence, the deviations contain important new information and have been a fruitful research area.

In contrast to the rapid progress in understanding the nature of afterglows, GRB itself has not yet been clear, i.e. the central engine. This is a very important problem. In fact, a viable model for the GRB central engine must address to the following problems:

\section{(i)Energy Budget}

The central engine must be able to release more than $10^{53}$ ergs or even $10^{54}$ ergs to radiation. The beaming factor can reduce the energy budget by a factor of 10-100, however, the conversion efficiency from particle kinetic energy to radiation energy likely increases the 
energy budget by a factor of 10. In fact, most merger models cannot release that much energy (Suen 2000, private communication).

\section{(ii)Baryon Contamination}

Another problem for existing central engine models is the baryon contamination. In order to explain the short time scales of the GRBs, the fireball must be relativistic. This requires the baryonic mass in the fireball must be less than $10^{-5}\left(E / 2 \times 10^{51} \mathrm{ergs}\right) M_{\odot}$. The failed supernova (e.g. Woosley 1993), collapsar of hypernova (e.g. Paczynski 1998), newly formed rapidly spinning and superstrong magnetic field neutron stars (e.g. Usov 1992; Kluzniak \& Ruderman 1998; Ruderman, Tao \& Kluzniak 2000) suffer from this problem. Models without baryon contamination problem, e.g. conversion of neutron star to strange star by accretion (Cheng \& Dai 1996; Dai \& Lu 1998), cannot explain GRB-SN association (Galama et al. 1998; Kulkarni et al. 1998). In fact, this is a dilemma of GRB-SN association unless some mechanisms, which can reduce the baryon contamination in supernova indeed exist (e.g. a two-step model proposed by Cheng \& Dai 2000).

\section{(iii)Birth Rate}

It must be larger than $10^{-6}$ per galaxy per year. The actual rate should be much larger than this if beaming exists.

\section{(iv) Various Time Scales}

The sub-structure of the light curves of GRBs can be as short as $10^{-3} \mathrm{~s}$ but the duration of GRBs can be as long as $10^{3} \mathrm{~s}$. The former suggests that the emission must be from very small volume and is normally interpreted as that the central engine must be a solar mass compact object, but the latter indicates that the energy release cannot take place in the gravitational time scale. In fact, there are a great variety of light curves in GRBs, which suggest that the energy release process cannot be a simple one. 


\section{(v)Acceleration and Microphysics}

The central engine must efficiently accelerate the ejecta to extremely relativistic. How do the collisionless shocks arise within the emitting region? How do these shocks accelerate the particles and enhance the magnetic fields?

Perhaps it is misleading to use the current observations to limit GRB models, i.e. GRB$\mathrm{SN}$ association, host galaxies etc. because they are all long duration GRBs. It is known that GRB durations scatter over six orders of magnitudes (from $1 \mathrm{~ms}$ to $1 \mathrm{ks}$ ), but a "typical" GRB lasts about $10 \mathrm{~s}$. The distribution of burst durations is bimodal, i.e., GRBs can be divided into two sub-groups: long bursts with $T_{90}>2 \mathrm{~s}$ and short bursts with $T_{90}<2 \mathrm{~s}$ (Piran 1999). The ratio of observed long bursts to short bursts is three to one. For example, HETEII, which was successfully launched recently and should be able to detect short duration bursts, may provide new information to constraint GRB models. In our opinion, we are far from understanding the nature of these mysterious GRB events. More information from future GRB missions in electromagnetic wave bands (for a review cf. Hurley 1999), in

gravitational wave bands (e.g. LIGO, VIRGO, TAMMA300 and GEO600, for a review of various gravitational detectors cf. Abramovichi et al. 1992; Bardachia et al. 1990; Throne 1997) and low energy neutrino detection, which can differentiate various merger models (Clark \& Eardley 1977), should help us to solve the puzzle of these important astrophysical phenomena.

We are happy to thank L.X. Cheng, S. Colgate, Z.G. Dai, K.Y. Ding, T. Harko, Y.F. Huang, Y. Lu, M. Ruderman, W.M. Suen, J.M. Wang, D.M. Wei for their useful conversations in various topics in GRBs. This work is partially supported by a RGC grant of the Hong Kong Government and the National Natural Science Foundation of China. 


\section{Figure Caption}

Fig. 1.- Typical light curves detected by BASTE (Greiner 1999)

Fig. 2.- Spatial Distribution of 1869 GRBs (Greiner 1999)

Fig. 3.- Spectra of two GRBs (Schaefer et al. 1998) 


\section{REFERENCES}

Abramovichi A. et al., 1992, Science, 256, 325

Akerlof, C., et al., 1999, Nature, 398, 400.

Alcock, C., Farhi, E., Olinto, A., 1986, ApJ 310, 261.

Andersen, M.I., et al., 1999, Science, 283, 2075.

Artymowicz, P., Lin, D.N.C., \& Wampler, E.J., 1993, ApJ, 409, 592

Band D.L. et al., 1996, ApJ, 458, 746

Bennett, D. P. \& Sun, H. R., 1996, ApJ, 458, 293

Bardachia C. et al., 1990, Nucl. Inst. A., 289, 518

Bethe, H.A., Brown, G.E., 1998, ApJ, 506, 780.

Blaes, O., Blandford, R., Goldreich, P., Madau, P., 1989, ApJ, 343, 839

Blandford, R.D., MaKee, C.F., 1976, Phys. Fluids, 19, 1130.

Blandford, R.D., Znajek, R.L., 1977, MNRAS, 179: 433.

Bloom, J.S. et al., 1999, Nature, 401, 453.

Brainerd, J. J., et al., 1995, ApJ, 441, L39

Briggs M.S., 1995, Ap\&SS, 231, 3

Carter, B., 1992, ApJ, 391, L67

Castro-Tirado, A.J., et al., 1999a, Science, 283, 2069.

Castro-Tirado, A., Gorosabel, J., 1999b, A\&AS, 138, 449. 
Cen R., 1998, ApJ, 507, L131

Cheng, K.S., Dai, Z.G., 1996a, Phys. Rev. Lett., 77, 1210.

Cheng, K.S., Dai, Z.G., Lu, T., 1998, Int. J. Mod. Phys. D, 7, 139.

Cheng, K.S., Dai, Z.G., 1999, astro-ph/9908248.

Cheng, K.S., Dai, Z.G., 2000, Astroparticle Physics, in press.

Cheng, K.S. \& Ding, K.Y., 1993, MNRAS, 262, 1037

Cheng, K.S., Wei, D.M., 1996, MNRAS, 283, L133.

Cheng, K.S. et al. , 1997, 1997 Pacific Rim Conference on Stellar Astrophysics, A.S.P. Conf. Ser. Vol. 138, eds. K.L. Chan, K.S. Cheng, H.P. Singh, 1998, 59.

Cheng, K.S., Wang, J.M., 1999, ApJ, 521, 502.

Cheng, K.S., Lu, Y., 2001, MNRAS, accepted

Cheng L.X., Ma Y.Q., Cheng K.S., Lu T., Zhou Y.Y., 1995,A\&A, 300, 746

Cheng L.X., Cheng K.S., 1996b, ApJ, 459, 79

Chevalier, R.A., Li, Z.-Y., 1999, ApJ, 520, L29.

Chevalier, R.A., Li, Z.-Y., 2000, ApJ, 536, 195.

Chiang, J., Dermer, C.D., 1999, ApJ, 512, 699.

Clark J.P.A., Eardley D., 1977, ApJ, 215, 311

Cohen E. et al., 1997, ApJ, 480, 330

Costa, E., et al., 1997, Nature, 387, 783. 
Dai, Z.G., Lu, T., 1998a, A\&A, 333, L87.

Dai, Z.G., Lu, T., 1998b, Phys. Rev. Lett., 81, 4301.

Dai, Z.G., Lu, T., 1998c, MNRAS, 298, 87.

Dai, Z.G., Huang, Y.F., Lu, T., 1999a, ApJ, 520, 634.

Dai, Z.G., Lu, T., 1999b, ApJ, 519, L155.

Dai, Z.G., Lu, T., 2000a, ApJ, 537, 803.

Dai, Z.G., Lu, T., 2000b, A\&A, in press, astro-ph/0005417.

Dai, Z.G., Lu, T., 2000c, ApJ, submitted (astro-ph/0002430).

Dai, Z.G., Gou, L.J., 2000d, ApJ, accepted (astro-ph/0010261).

Dai, Z.G., 2000e, Proc. First KIAS International Workshop on Astrophysics: Explosive Phenomena in Compact Astrophysical Systems, Seoul, Korea; 24-27 May 2000, astro$\mathrm{ph} / 0008304$.

Dai, Z.G., Huang, Y.F. \& Lu T., 2000f, MNRAS (Letters), submitted.

Davies M.B., Benz W., Piran T., Thielemann F.K., 1994, ApJ, 431, 742

Dermer, C.D., 1992, Phys.Rev. Lett, 68, 1799

Ding, K.Y., Cheng, K.S., 1997, MNRAS, 286, 671

Duncan, R.,Thompson, C., 1992, ApJ, 392, L9.

Eichler D., Livio M., Piran T., Schramm DX, 1989, Nature, 340, 126

Fenimore E.E. et al., 1988, ApJ, 335, L71 
Ford L.A. et al., 1995, ApJ, 439, 307

Frail, D.A. et al., 1997, Nature, 389, 261.

Frail, D.A. et al., 2000, ApJ, 534, 559.

Fruchter, A. et al., 1999, ApJ, 513, L13.

Galama, T.J., et al., 1997, Nature, 387, 497.

Galama, T.J., et al., 1998, Nature, 395, 670.

Galama, T.J., et al., 1999, Nature, 398, 394.

Galama, T.J., et al., 2000, ApJ, 536, 185.

Glendenning, N.K., 1992, Phys. Rev. D., 46, 1274.

Goodman, J., 1986, ApJ, 308, L47.

Gorosabel, J., Castro-Tirado, A. J., 1997, ApJ, 483, L83.

Gou, L.J. et al., 2000, A\&A, accepted, astro-ph/0010244.

Greiner, J., 1999, Mem. Soc. Astron. Ital., 70, 891

Groot, P.J. et al., 1997, IAUC, No.6584.

Harding, A.K., 1991, Phys. Rep., 206, 327.

Harko, T. and Cheng, K.S., 2000, Phys. Lett. A, 266, 249

Hartmann, D.H., Narayan, R., 1996, ApJ, 464, 226

Haensel, P., Paczyński, B., Amsterdamski, P., 1992,ApJ, 375, 209. 
Halzen, F., 1998, Lectures on Neutrino Astronomy: Theory and Experiment, astroph/9810368.

Haswell C.A., Tajima T., Sakai J.-I. 1992, ApJ, 401, 495.

Hjorth, J., et al., 1999, Science, 283, 2073.

Hogg, D.W., Fruchter, A., 1999, ApJ, 520, 54

Huang, Y.F., Lu, T., 1997a, Chin. Phys. Lett., 14, 314.

Huang, Y.F., Lu, T., 1997b, A\&A, 325, 189-194.

Huang, Y.F., Dai, Z.G., Lu, T., 1998a, A\&A, 336, L69.

Huang, Y.F., Dai, Z.G., Wei, D.M., Lu, T., 1998b, MNRAS, 298, 459.

Huang, Y.F., Dai, Z.G., Lu, T., 1999a, MNRAS, 309, 513.

Huang, Y.F., Dai, Z.G., Lu, T., 1999b, Chin. Phys. Lett., 16, 775, astro-ph/9906404.

Huang, Y.F., Gou, L.J., Dai, Z.G., Lu, T., 2000a, ApJ, 543, 90.

Huang, Y.F., Dai, Z.G. and Lu, T., 2000b, MNRAS, 316, 943.

Huang, Y.F., Dai, Z.G. and Lu, T., 2000c, A\&A, 355, L43.

Huang, Y.F., Dai, Z.G. and Lu, T., 2000d, Chin. Phys. Lett., 17, 778.

Hunter, G.J., 1984, in Woosley S.E. et al. Eds, AIP Conf. Proc. High Energy Transient in Astrophysics. Am Inst. Phys., New York, P.373.

Hurley, K., et al., 1997, ApJ, 479, L113

Hurley K., 1999, A\&AS, 138, 553 
Katz, J., 1994, ApJ, 422, 248.

Katz, J., Canel, L.M.,1996, ApJ, 471, 527

Klebesadel, R.W., Strong, I.B., Olson, R.A., 1973, ApJ, 182, L85.

Kluźniak, W., Ruderman, M., 1998, ApJ, 505, L113.

Kolatt, P., Piran, T., 1996, ApJ, 467, L41

Kouveliotou, C. et al., 1993, ApJ, 413, L101.

Krolik, J.H., Pier, E.A., 1991, ApJ, 373, 277.

Kulkarni, S. R., et al., 1998, Nature, 395, 663.

Kulkarni, S. R., et al., 1999a, Nature, 398, 389.

Kulkarni, S. R., et al., 1999b, ApJ, 522, L97

Kulkarni, S.R. et al., 1999c, ApJ, 522, L97.

Lee, H.K., Wijers, R.A.M.J., Brown, G.E., 2000, Phys. Rep., 325, 83.

Li, H., Dermer, C.D., 1992, Nature, 359, 514

Lyne, A.G., Lorimer, D.R., 1994, Nature, 369, 127

Lu, T., 1997, 1997 Pacific Rim Conference on Stellar Astrophysics, A.S.P. Conf. Ser. Vol. 138, eds. K.L. Chan, K.S. Cheng, H.P. Singh, 1998, 215.

Lu, T., invited talk presented at the 1999 Pacific Rim Conference on Stellar Astrophysics, astro-ph/0002178.

Lu, T., 2000, Proc. 3rd Joint Meeting of Chinese Physicists Worldwide, August, Hong Kong, China. 
Lu, Y., Cheng, K.S., Zhao, G., Yang, L.T., 2000, Chin. Phys., Lett., 17, 73.

Lu, Y., Cheng, K.S., Zhao, G., 2000, PASJ, 52, 711

Manchester, R.N., 1999, 1999 Pacific Rim Conference on Stellar Astrophysics, eds. Cheng, K.S. et al., Kluwer, Netherland.

Mao, S., Paczynski, B., 1992, ApJ, 388, L45

Marani, G. F., Nemiroff, R. J., Norris, J. P., Bonnell, J. T., 1997, ApJ, 474, 576.

Mazets E. et al., 1980, Sov. Astron. Lett., 6, 372

Mazets, E.P., et al., 1981, Nature, 290, 378

Meegan, C. A., et al., 1992, Nature, 355, 143.

Meegan C.A. et al., 1995, ApJ, 434, 552

Mészáros, P., Rees, M.J., 1992, MNRAS, 257, 29.

Mészáros, P., Rees, M.J., 1997a, ApJ, 476, 232.

Mészáros, P., Rees, M.J., 1997b, ApJ, 482, L29.

Mészáros, P., 1999, astro-ph/9904038.

Mitra, A., 1998, ApJ, 492, 677.

Moderski, R., Sikora, M., Bulik, T., 2000, ApJ, 529, 151.

Murakami T. et al., 1988, Nature, 335, 234

Narayan R., Piran T., Shemi A., 1991, ApJ, 379, L17

Norris J.P. et al., 1986, ApJ, 301, 213 
Norris, J.P. et al., 1994, ApJ, 424, 540.

Paczyński, B., 1986, ApJ, 308, L51.

Paczyński, B., Xu, G., 1994, ApJ, 427, 708.

Paczyński, B., 1998, ApJ, 494, L45.

Palmer D.M. et al., 1994, ApJ, 433, L77

Panaitescu, A., Mészáros, P., Rees, M.J., 1998, ApJ, 503, 314.

Panaitescu, A., Mészáros, P., 1999, ApJ, 526, 707.

Pendleton G.N. et al., 1995, AAS, 86, 501

Piran, T., 1999, Phys. Rep., 314, 575.

Piran, T., 2000, Phys. Rep., 333, 529.

Podsiadlowski, P., Rees, M.J., Ruderman, M., 1995, MNRAS, 273, 755

Popham, R., Woosley, S.E., Fryer, C., 1999, ApJ, 518, 356

Pugliese, G., Falcke, H., Biermann, P.L., 2000, A\&A, 358, 409.

Quashnock, J.M., Lamb, D.Q., 1993, MNRAS, 265, L59

Ramaty, R.S. et al., 1980, Nature, 287, 122.

Reichart, D.E., 1999, ApJ, 521, L111.

Rees, M.J., Mészáros, P., 1992, MNRAS, 258, 41.

Rees, M.J., Mészáros, P., 1994, ApJ, 430, L93.

Rees, M.J., Mészáros, P., 1998, ApJ, 496, L1. 
Rhoads, J., 1997, ApJ, 487, L1.

Rhoads, J., 1999, ApJ, 525, 737, astro-ph/9903399).

Ruderman, M., Cheng, K.S., 1988, ApJ, 335, 306

Ruderman, M., Tao, L., Kluźniak, W., 2000, ApJ, 542, 243.

Ruffert M., Janka H.T., 1998, A\&A, 338, 535.

Sari, R., Piran, T., 1995, ApJ, 455, L143.

Sari, R., Piran, T., Narayan, R., 1998, ApJ, 497, L17.

Sari, R., Piran, T., Halpern, J.P., 1999, ApJ, 519, L17.

Schaefer, B.E., et al., 1998, ApJ, 492, 696.

Schartel, N., Andernach, H., Greiner, J., 1997, A\&A, 323, 659

Sedov, L., 1969, Similarity and Dimensional Methods in Mechanics (Academic, New York), Chap.IV.

Taylor, J.H. \& Weisberg, J.M., 1989, ApJ, 345,434.

Taylor, G. et al., 1997, Nature, 389, 263.

Tinney, C. et al., 1998, IAU Circ. 6896.

Thorne, K.S., 1997, Rev. Mod. Astron. 10, 1

Usov V.V.1 1992, Nature, 357, 472-

Van Paradijs, J., et al., 1997, Nature, 386, 686.

Van Paradijs, J., et al., 2000, ARA\&A, 38, 379. 
Vietri, M., 1997, ApJ, 488, L105.

Wang, L., Wheeler, J.C., 1988, ApJ, 504, L87

Wang, X.Y., Dai, Z.G. and Lu, T., 2000a, MNRAS, 317, 170.

Wang, X.Y., Dai, Z.G., Lu, T., Wei, D.M., Huang, Y.F., 2000b, A\&A, 357, 543.

Wang, X.Y., Dai, Z.G. and Lu, T., 2000c, ApJL, accepted, astro-ph/0010320.

Wang, X.Y., Dai, Z.G., Lu, T., 2000d, MNRAS, in press, astro-ph/9906062.

Wang, W., Dai, Z.G., 2000e, Chin. Phys. Lett., submitted.

Waxman, E., 1997a, ApJ, 485, L5.

Waxman, E., Bahcall, J.N., 1997b, Phys. Rev. Lett., 78, 2292.

Waxman, E., Bahcall, J.N., 2000, ApJ, 541, 707.

Wei,D.M., Cheng,K.S., 1997, MNRAS, 289, 681

Wei, D.M., Cheng, K.S.,1997, MNRAS, 290,107

Wei, D.M., Lu, T., 1996, ApSS, 235, 289

Wei, D.M., Lu, T., 1997, A\&A, 323, 312.

Wei, D.M., Lu, T., 1998a, ApJ, 499, 754.

Wei, D.M., Lu, T., 1998b, ApJ, 505, 252.

Wei, D.M., Lu, T., 2000a, A\&A, 360, L13.

Wei, D.M., Lu, T., 2000b, ApJ, 541, 203.

Wei, D.M., Lu, T., 2000c, astro-ph/9912063. 
Wheeler, J.Craig, 1999,Conference summary: SNe \& GRBs, published in: the Largest Explosions Since the Big Bang: Supernovae and Gamma-Ray Bursts, eds. M. Livio, K. Sahu, N. Panagia, astro-ph/9909096.

Wijers, R.A.M.J., Rees, M.J., Mészáros, P., 1997, MNRAS, 288, L51.

Woosley S.E., 1993, ApJ, 405, 273

Woosley, S.E., Macfadyen, A.I., Heger, A., 1999, published in: "Supernovae and Gamma-Ray bursts", eds. M. Livio, K. Sahu, N. Panagia, astro-ph/9909034.

Zurek, W.H., Siemiginowska, A. \& Colgate, S.A., 1994, ApJ, 434, 46 